\title{
DENSE SOLID-LIQUID OFF-BOTTOM SUSPENSION DYNAMICS: SIMULATION AND EXPERIMENT
}

\author{
A. Tamburini ${ }^{\text {a }}$, A. Cipollina ${ }^{\text {a }}$, G. Micale ${ }^{\text {a }}$, M. Ciofalo ${ }^{\text {b }}$, A. Brucato ${ }^{\text {a }}$ \\ ${ }^{a}$ Dipartimento di Ingegneria Chimica dei Processi e dei Materiali \\ Università di Palermo, Viale delle Scienze Ed. 6, 90128 Palermo (ITALY); \\ e-mail: a.tamburini@dicpm.unipa.it; micale@dicpm.unipa.it \\ ${ }^{b}$ Dipartimento di Ingegneria Nucleare \\ Università di Palermo, Viale delle Scienze Ed. 6, 90128 Palermo (ITALY)
}

\begin{abstract}
Dense solid-liquid off-bottom suspension inside a baffled mechanically agitated stirred tank equipped with a standard Rushton turbine is investigated. Dynamic evolution of the suspension from start up to steady state conditions has been inspected by both visual experiments and computational fluid dynamics. A classical Eulerian-Eulerian Multi Fluid Model along with the "homogeneous" k- $\varepsilon$ turbulence model is adopted to simulate suspension dynamics. In these systems the drag inter-phase force affects both solids suspension and distribution. Therefore, different computational approaches are tested in order to compute this term. Simulation results are compared with images acquired on the real system and a good agreement is found.
\end{abstract}

Key words: Mixing; Suspension; Startup; Drag; Solid Phase Viscosity; Solid-Liquid.

\section{INTRODUCTION}

Solid-liquid operations are frequently encountered in the chemical and process industries: there is indeed a number of industrial operations involving the suspension of solid particles in a liquid phase inside mechanically stirred vessels. Because of the high industrial relevance a significant effort has been devoted to better understanding the phenomena involved.

In recent years Computational Fluid Dynamics (CFD) is increasingly employed as a fundamental tool to critically analyze solid-liquid flows and related phenomena. Two different approaches exist to model such multiphase systems: Eulerian-Eulerian and EulerianLagrangian models $[1,2,3,4,5]$.

Eulerian-Eulerian models consider both the continuum and the dispersed phase as interpenetrating continua. Conversely in the Eulerian-Lagrangian approach, the fluid is treated as a continuum by solving the Navier-Stokes equations, either averaged or not, while the dispersed phase is treated by tracking a large number of particles through the calculated flow field.

Eulerian-Eulerian approaches involving the classical Multi Fluid Model (MFM) are often preferred to the Eulerian-Lagrangian ones because of some important reasons: simplicity, lower computational requirements and their capability to deal with high solid loading conditions (dense suspensions). 
Recently, various simulation attempts have been made to study the behaviour of dense suspensions inside stirred tanks $[6,7,8,9]$ showing a good agreement between experiment and numerical predictions for steady state systems. No works have so far been addressed to investigate transient startup condition.

Aim of the present work is the numerical prediction of the transient dynamic evolution of a dense solid-liquid suspension inside a non-standard geometry stirred tank from start-up to steady state conditions. Computational results were qualitatively compared with experimental data in order to validate the modeling approach adopted.

It is worth noting that dense suspensions require a correct modeling of both solid-liquid and particle-particle interactions. Several studies have dealt with the interphase drag term or simply the drag coefficient $[4,8,9,10,11]$ accounting for the solid volume fraction or turbulence effects. Fewer efforts have been devoted so far to the problem of particle-particle interactions $[5,10,11]$.

\section{PHYSICAL SYSTEM AND SIMULATION PROCEDURES DESCRIPTION}

\subsection{Experimental apparatus}

The experimental system consisted of a cylindrical, flat-bottomed, baffled tank with vessel diameter $\mathrm{T}=0.19 \mathrm{~m}$ and liquid height equal to $1.5 \cdot \mathrm{T}$, as depicted in Fig.1. A standard six bladed Rushton turbine was used in the suspension experiments. A close off-bottom impeller clearance was chosen in order to ensure a "single-loop" flow configuration [12]. The agitator speed here investigated $(380 \mathrm{rpm})$ was sufficient to get fully suspended conditions at steady state. The liquid level $(\mathrm{H})$ was higher than usual $(\mathrm{H}=1.5 \mathrm{~T}$, instead of $\mathrm{H}=\mathrm{T})$ in order to widen the range of stirrer speed velocities for which the clear liquid existence is clearly observed.

Deionised water and silica particles with diameter ranging between 212 and $250 \mu \mathrm{m}$ were employed. Solid weight fraction was $21.5 \% \mathrm{w} / \mathrm{w}(9.6 \% \mathrm{v} / \mathrm{v})$. The particle bed laying on the bottom under no agitation conditions was found to have a void fraction of $38 \%$.

Transient dynamic evolution of the off-bottom suspension was recorded by a high resolution digital camera acquiring 19 frames per second.
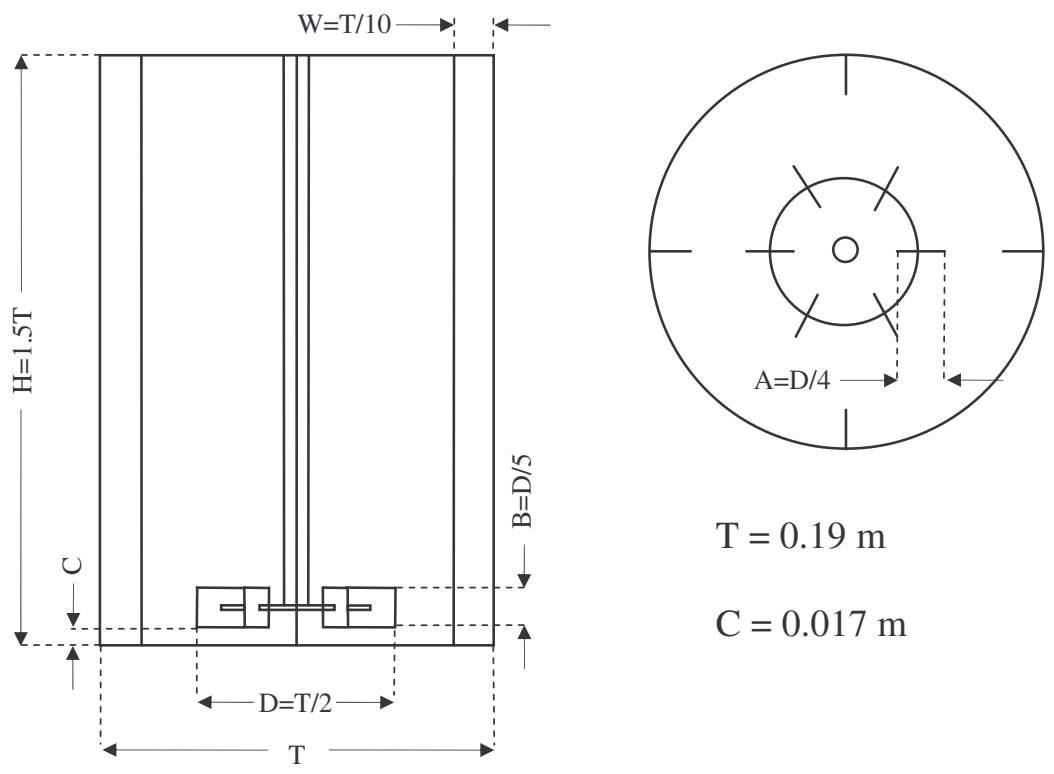

$$
\begin{aligned}
& T=0.19 \mathrm{~m} \\
& \mathrm{C}=0.017 \mathrm{~m}
\end{aligned}
$$

Figure 1. Sketch of the experimental apparatus 


\subsection{Computational approaches and numerical simulations}

For all CFD simulations, the Eulerian-Eulerian "Multi Fluid Model" (MFM), implemented in the commercial code CFX release 4.4, developed by AEA Technology, was used. This model solves the continuity and momentum equations for a generic multi-phase system and therefore allows the determination of separate flow field solutions for each phase simultaneously. The particle phase was treated as a separate dispersed phase, occupying disconnected regions of space in the continuous liquid phase.

A $24 \times 69 \times 32$ (azimuthal $\times$ axial $\times$ radial) finite volume structured grid was used to discretize the domain region. In the azimuthal direction, thanks to symmetry, only half of the vessel was simulated, with 24 equally-spaced angular subdivisions. The other half of the tank was simulated imposing periodic boundary conditions along the azimuthal direction. The computational grid adopted has a smaller cell spacing in the lower part of the vessel, near the impeller, where the largest gradients of flow quantities are expected. A finer grid $48 \times 138 \times$ 64 was also used to check whether results were affected by grid dependence.

Turbulent solid-liquid flow was computed by a standard "homogeneous" k- $\varepsilon$ turbulence model that has been found to provide a fair representation of solids distribution in high density stirred reactors [7, 9].

A sliding cylindrical surface was set just outside the impeller region in order to model impeller-baffles relative motion.

The following table shows the physical properties of the two phases as set in the simulation.

Table 1. Physical parameters of the two phases modelled

\begin{tabular}{lcc}
\hline & Liquid (water) & Solid (silica) \\
\hline Density $\left[\mathrm{kg} / \mathrm{m}^{3}\right]$ & 1000 & 2580 \\
\hline Viscosity $[\mathrm{Pa} \cdot \mathrm{s}]$ & $10^{-3}$ & $10^{-3}$ \\
\hline Particle diameter $[\mathrm{m}]$ & - & $2.31 \cdot 10^{-4}$ \\
\hline Mean volumetric fraction & 0.904 & 0.096 \\
\hline Volumetric fraction at max packing & 0.38 & 0.62 \\
\hline
\end{tabular}

Interactions between the two phases were modelled only by interphase drag terms inside momentum equations (two-way coupling). A choice of approaches were used to correctly model the influence of slip velocity, turbulence and solid volume fraction on the interphase drag term.

The effect of high particle concentrations on drag force were taken into account by adopting the following equations for the interphase drag force:

- for low solid fractions $\left(0<r_{\beta}<r_{\beta \_ \text {min }}\right)$

$$
\vec{F}_{D_{\alpha \beta}}=C_{\alpha \beta}\left(\vec{U}_{\beta}-\vec{U}_{\alpha}\right)=\left[\frac{3}{4} \frac{C_{D}}{d_{p}} r_{\beta} \rho_{\alpha}\left|\vec{U}_{\beta}-\vec{U}_{\alpha}\right| *\left(1-r_{\beta}\right)^{-1.65}\right]\left(\vec{U}_{\beta}-\vec{U}_{\alpha}\right)
$$

where subscripts $\alpha$ and $\beta$ indicate the liquid and solid phases respectively, $d_{p}$ is particle diameter, $\rho$ is density, $U$ is mean velocity, $r$ is volume fraction, and $C_{D}$ is the drag coefficient. Eq.(1) is utilized if the solid volume fraction is lower than a fixed value $r_{\beta_{-} \min }$; in practice with this equation the drag inter-phase term is calculated by the standard $C_{\alpha \beta}$ formulation along with Gidaspow's correction for dense particle effects [11]; 
- for solid volumetric fractions variable from $r_{\beta_{-} \max }$ to the maximum value $r_{\beta}$ packed, eq.(2) is used $\left(r_{\beta_{-} \max }<r_{\beta}<r_{\beta \_ \text {packed }}\right)$

$$
\vec{F}_{D_{\alpha \beta}}=C_{\alpha \beta}\left(\vec{U}_{\beta}-\vec{U}_{\alpha}\right)=\left[150 \frac{r_{\beta}^{2} \mu_{\alpha}}{\left(1-r_{\beta}\right) d_{p}^{2}}+1.75 \frac{r_{\beta} \rho_{\alpha}\left|\vec{U}_{\beta}-\vec{U}_{\alpha}\right|}{d_{p}}\right]\left(\vec{U}_{\beta}-\vec{U}_{\alpha}\right)
$$

Here $\mu$ is viscosity and $C_{\alpha \beta}$ is obtained in practice via the well known Ergun equation, typically used to describe closely packed fixed-bed systems;

- for intermediate volume fractions $\left(r_{\beta_{-} \min }<r_{\beta}<r_{\beta_{-} \max }\right)$

$$
\vec{F}_{D_{\alpha \beta}}=\left[C_{\alpha \beta}\left(r_{\beta_{-} \min }\right)+\frac{C_{\alpha \beta}\left(r_{\beta_{-} \max }\right)-C_{\alpha \beta}\left(r_{\beta_{-} \min }\right)}{r_{\beta_{-} \max }-r_{\beta_{-} \min }}\left(r_{\beta}-r_{\beta_{-} \min }\right)\right]\left(\vec{U}_{\beta}-\vec{U}_{\alpha}\right)
$$

i.e. a linear interpolation was employed thus avoiding any discontinuity in calculating $C_{\alpha \beta}$ by the two previous expressions. On the basis of Eq.(1) and Eq.(2) validity fields, $r_{\beta} \_$min and $r_{\beta \_\max }$ were set to 0.35 and 0.45 respectively, as long as a monotonic dependence of $C_{\alpha \beta} v s r_{\beta}$ was obtained, as shown in Fig.2a. Where the slip velocity was so large that a non-monotonic dependence would have resulted, the validity range for Eq.3 was suitably enlarged in order to avoid it, as shown in Fig.2b.
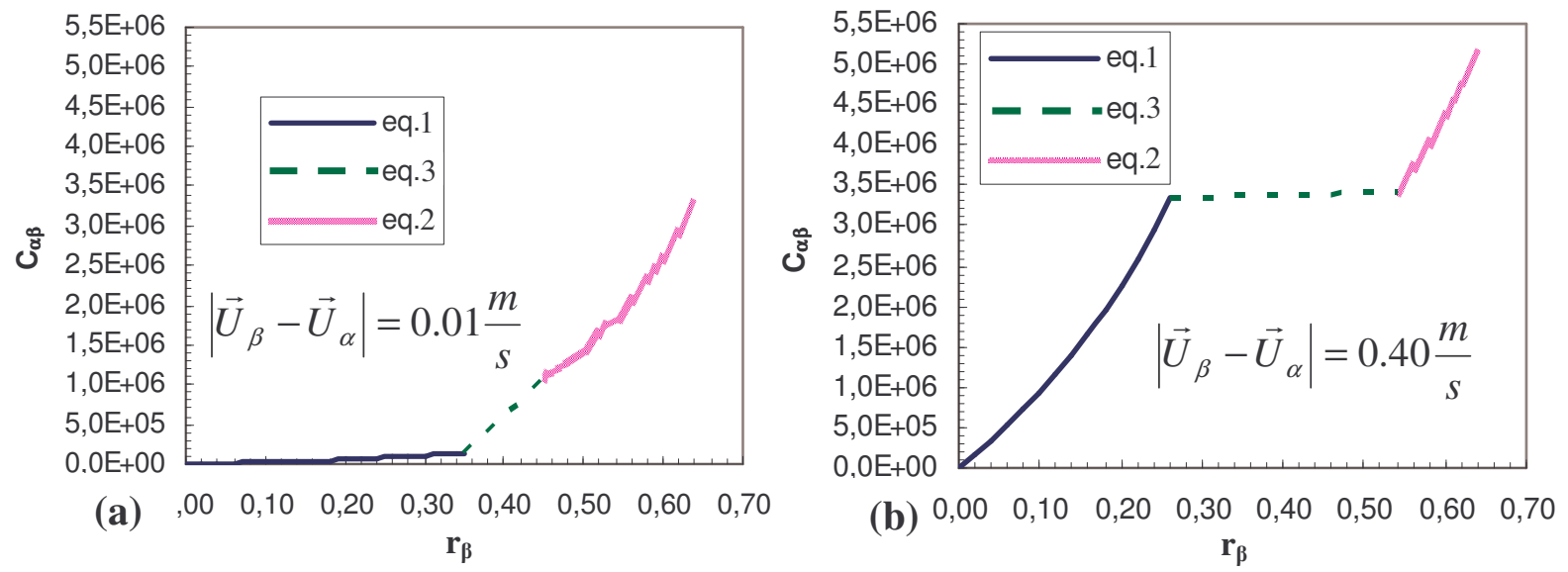

Figure 2. The complete $C_{\alpha \beta}\left(r_{\beta}\right)$ function at two different slip velocity values.

For the drag coefficient ( $C_{D}$ ) calculation, different approaches were adopted.

1) The simplest one computes it as for a single particle settling at its terminal velocity $(\sim 3$ $\mathrm{cm} / \mathrm{s}$ ) in a quiescent fluid on the basis of the general equation for transition region (for the particle-fluid system here employed $C_{D}=6.01$ ). This approach will be called as fixed$C_{D}$.

2) Another approach consider a $C_{D}$ variable in each cell in relation to the slip velocity: here $C_{D}$ is calculated by the Clift et al correlation (eq. 4) where cell slip velocity was used for the Reynolds number calculation. This approach will be called as slip- $C_{D}$. 


$$
C_{D}=\frac{24}{\operatorname{Re}_{p}}\left(1+0.2 \operatorname{Re}_{p}^{0.63}\right)
$$

3) In the last case, called turb- $C_{D}$, free-stream turbulence influence upon drag inter-phase force was accounted for by implementing Brucato et al correlation [14]

$$
C_{D, \text { turb }}=C_{D O}\left[1+8.67 \times 10^{-4}\left(\frac{d_{p}}{\lambda}\right)^{3}\right]
$$

where $C_{D O}$ was computed by equation (4), $d_{p}$ is particle diameter and $\lambda$ is the well known Kolmogorov length scale.

At the beginning of each simulation all particles were placed on the tank bottom with a initial volume fraction of $0.62 \mathrm{v} / \mathrm{v}$, corresponding to the experimentally assessed maximum packing.

An algorithm, named excess solid volume correction [13], was implemented inside an iterative procedure at the end of each SIMPLEC iteration in order to avoid exceeding this value. This iterative use of the algorithm acts as a posteriori correction redistributing the overpacking solids before the simulation goes on.

Simulations were conducted using two different time steps. A finer $\mathrm{TS}_{1}$ was used during the first ten revolutions to better follow the initial system evolution, while a ten fold time step $\mathrm{TS}_{2}$ was utilized for simulating the system from the $10^{\text {th }}$ impeller revolution on. $\mathrm{TS}_{2}$ corresponds to the time impeller needs to rotate by an angle equal to the azimuthal length of a single computational cell. Therefore each of the first ten revolutions requires 480 time steps, while each of the last ninety requires 48 time steps.

\section{RESULTS AND DISCUSSION}

Simulation results and visual experimental information are reported in Fig.3. Each row shows pictures of the suspension after 1,2,5,10,20,50 complete impeller revolutions respectively, that is from a still almost motionless condition to steady-state. As a matter of fact no appreciable variations in suspension height and solid distribution were visible after the $50^{\text {th }}$ revolution.

The first and the last columns are experimental images of the suspension, acquired by placing the camera at different tilt angles. All computational images (columns 2,3,4,5 of Fig.3) show solid volume fraction distributions on a diametral vertical plane midway between subsequent baffles; the legend range sweeps from 0 to twice the value of the mean particle volumetric fraction (i.e. $0.192 \mathrm{v} / \mathrm{v}$ ). All simulation results were obtained, using Eqs. $(1,2,3)$ for computing the drag force, while the drag coefficient model was the fixed- $C_{D}$ for column 2 and 3 , the slip- $C_{D}$ for column 4 , and the turb- $C_{D}$ for column 5 . In all computational results of rows (a) and (b) a decrease in suspension height in the central part of the tank upon the impeller disk is visible, thus generating a partial and a total impeller uncovering, respectively. This phenomenon is appreciable observing the experimental tilted images (6a and $6 \mathrm{~b}$ ) too. Comparison of other subsequent frames shows a good level of agreement between predicted and observed suspension height, thus a good prediction of the entire transient solid distribution was obtained.

No significant variations among the chosen computational approaches are appreciable, each of them manages to correctly follow the transient, apart from a little increase of suspension height when the turb- $C_{D}$ method is adopted. 


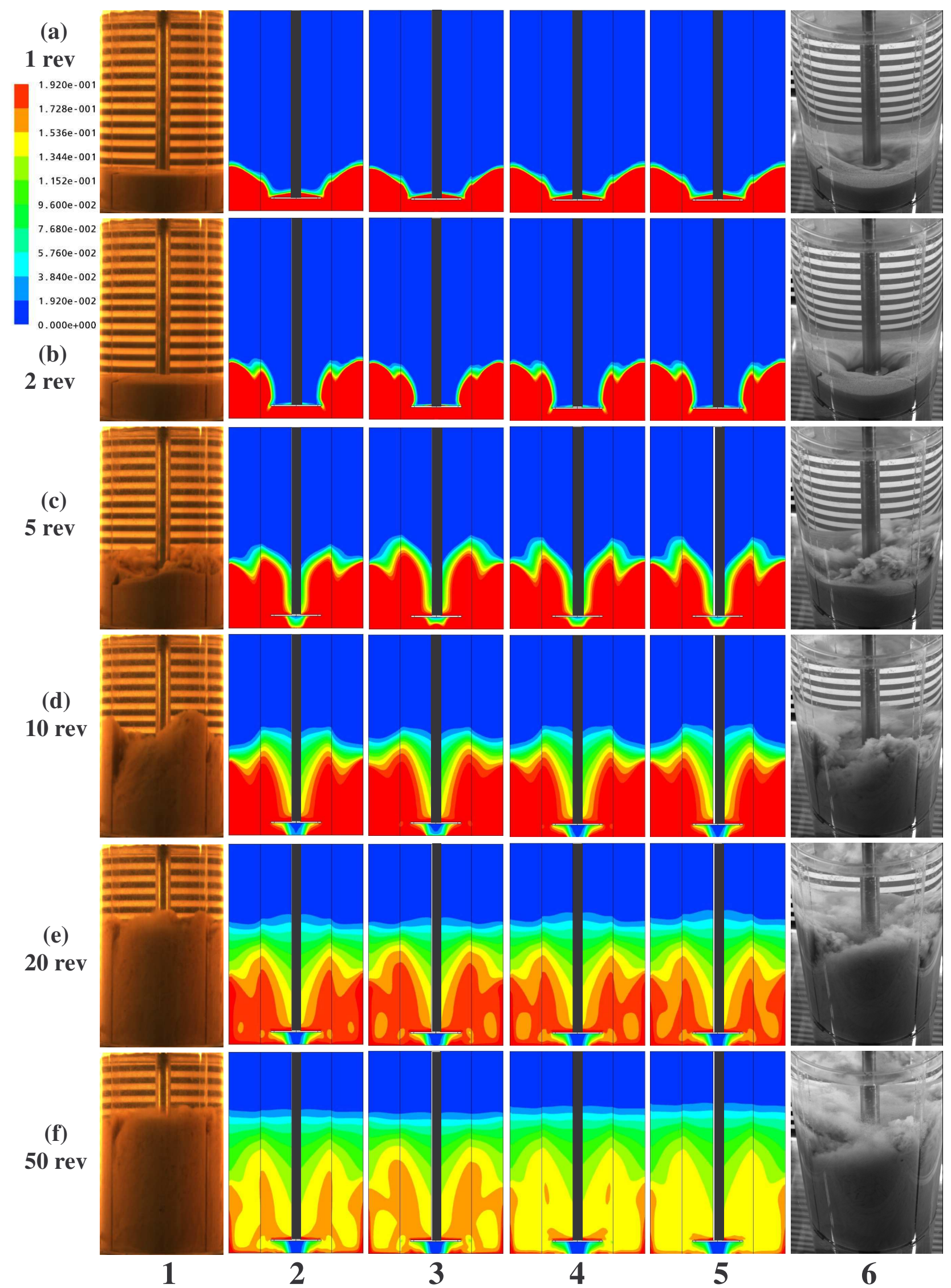

Figure 3. Solid volume fraction distribution during start-up transitory of an off-bottom suspension: comparison between simulations and experiments. 1) experiment, frontal view; 2) simulated distribution in a vertical diametral plane with fixed- $C_{D}$; 3) with fixed- $C_{D}$ and the finer computational grid; 4) with slip- $C_{D}$; 5) with turb- $C_{D}$; 6) experiment, inclined frontal view. 
Computational data of column 3 were obtained by using the finer grid and they provide very similar results to those of column 2, thus suggesting limited grid-dependence, as far as particle distribution is concerned. This is further confirmed by the steady state axial profiles of solids concentration depicted in Fig.4.

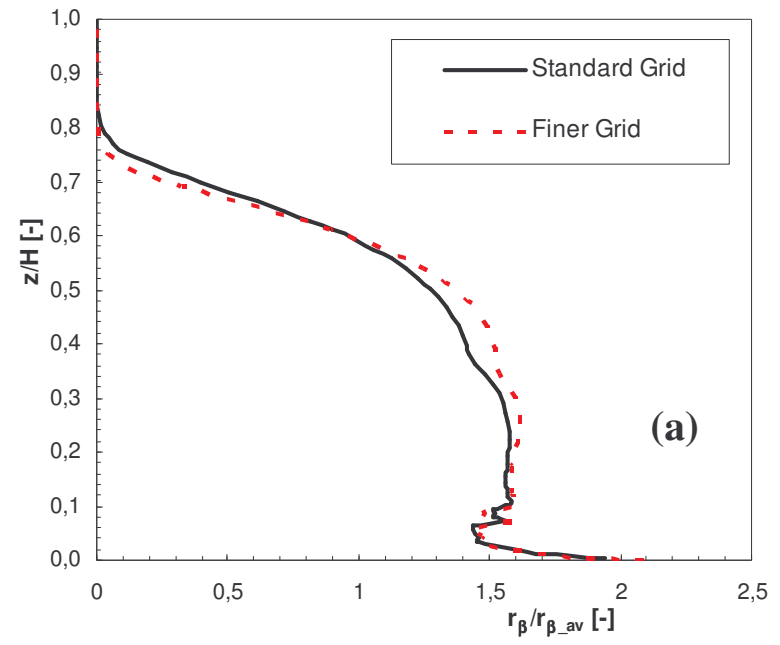

Figure 4. Steady state radially averaged axial profile of solid particles concentration on a vertical plane midway two subsequent baffles: comparison between the two different grids.

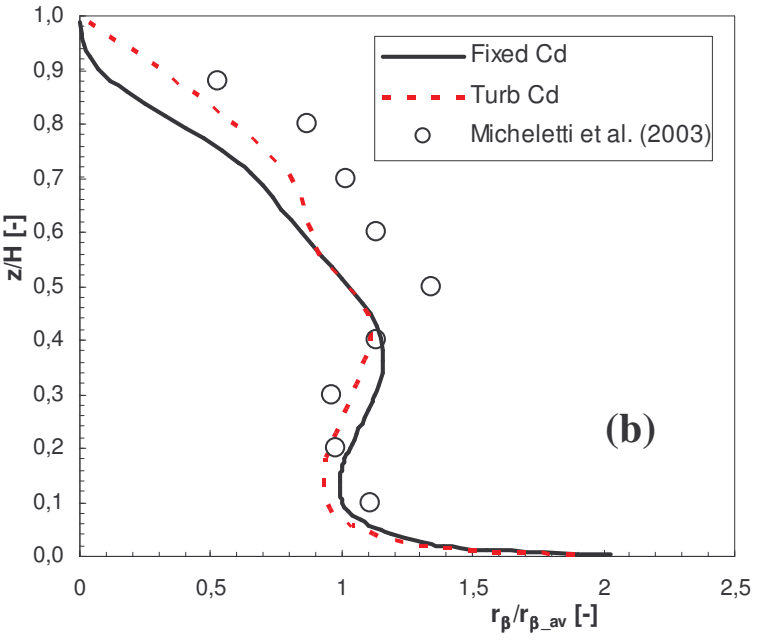

Figure 5. Steady state local axial profile of solid particles concentration at 800 $\mathrm{rpm}$ at $\mathrm{r} / \mathrm{T}=0.35$ and midway between subsequent baffles

In order to further test model reliability, in Fig.5 experimental data by Micheletti et al [15] are compared with relevant CFD predictions. Simulation predictions show a fair agreement with experimental data obtained at $\mathrm{N}=800 \mathrm{rpm}$. The turb $-C_{D}$, simulation shows somewhat better agreement than the fixed- $C_{D}$ simulation, though there seems to be room for further model improvement.

Velocity vector plots, not shown here for the sake of brevity, clearly follow the suspension evolution: higher values of velocities are always limited to the tank zone where particles are present. Indeed, they are located in the lower part of the vessel near the impeller during the first revolution and successively, during the subsequent revolutions, they develop towards the upper part of the tank.

The good agreement observed between experiment and simulations with the proposed simple modelling methods suggests that suspension of dense solid-liquid systems in stirred tank is a phenomenon mainly controlled by macroscopic effects such as gravity, centrifugal and inter-phase drag forces.

\section{CONCLUSIONS}

A CFD methodology has been developed to simulate the transient behaviour of dense solid-liquid suspensions in a stirred vessel during start-up. The Eulerian-Eulerian Multi Fluid Model along with the standard homogeneous k- $\varepsilon$ turbulence model was adopted. The sliding grid algorithm was used to simulate the impeller rotation in the fully baffled tank. Several equations for inter-phase drag force have been implemented to account for its dependence on solid volumetric fraction. Different approaches have been presented for drag coefficient 
calculation. Finally, a grid dependence investigation has been made by discretizing the domain by a finer computational grid.

Comparing experimental frames with relevant computational pictures an high level of agreement has been observed, independently of the adopted approach, thus suggesting suspension of high solid loading with liquids in a stirred fully baffled tank depends predominantly on inter-phase drag and body forces.

\section{REFERENCES}

1. Micale G., Montante G., Grisafi F., Brucato A., Godfrey J., 2000. "CFD simulation of particle distribution in stirred vessels", Trans. IChemE, Part A, Chem.Eng. Res. \& Des., 78, 435-444.

2. Decker S., Sommerfeld M., 1996, "Calculation of particles suspension in agitated vessels with the Euler-Lagrange approach", I. Chem. E. Symp. Ser., 140, 71-82 IChem, Rugby.

3. Montante G., Micale G., Magelli F. and Brucato A., 2001, "Experiments and CFD predictions of solid particle distribution in a vessel agitated with four pitched blade turbines", Trans. IChemE, Part A, Chem. Eng. Res. \& Des., 79, 1-6.

4. Montante G., Magelli F., 2007, "Mixed Solids Distribution in Stirred Vessels: Experiments and Computational Fluid Dynamics Simulations", Ind. Eng. Chem. Res., 46 , 2885-2891.

5. Derksen J. J., 2003, "Numerical Simulation of Solid Suspension in a Stirred Tank", AICHE Journal 49, 2700-2714.

6. Brucato A., Micale G., Montante G., Scuzzarella A., 2002, "Experimental Investigation and CFD Simulation of Dense Solid-Liquid Suspensions in a Fully Baffled Mechanically Stirred Tank", Proc. 10 $0^{\text {th }}$ Workshop on two-phase flow predictions, (Merseburg, Germany, 9-12 April), pp 255-264.

7. Micale G., Grisafi F., Rizzuti L., and Brucato A., 2004, "CFD Simulation of Particle Suspension Height in Stirred Vessels", Chem. Eng. Research and Design, 82(A9), 12041213.

8. Khopkar A.R., Kasat G.R., Pandit A.B., and Ranade V.V., 2006, "Computational Fluid Dynamics Simulation of the Solid Suspension in a Stirred Slurry Reactor", Ind. Eng. Chem. Res., 45, 4416-4428.

9. Montante G., Magelli F., 2005, "Modelling of Solids Distribution in Stirred Tanks: Analysis of Simulation Strategies and Comparison with Experimental Data", Int. Jour. Comp. Fluid Dyn., 19, ํ3, 253-262.

10. Ochieng A., Onyango M. S., 2008, "Drag Models, Solids Concentration and Velocity Distribution in a Stirred Tank", Powder Technology, 181, 1-8.

11. Gidaspow D., 1994, Multiphase flow and fluidization, Academic Press, San Diego.

12. Montante G., Lee KC, Brucato A., Yianneskis M., 1999, "An Experimental Study of Double-to-Single-Loop Transition in Stirred Vessel", Canadian Journal of chemical engineering, 77, 649-659.

13. Lettieri P., Cammarata L., Micale G., and Yates J., 2003, "CFD Simulations of Gas Fluidized Beds Using Alternative Eulerian-Eulerian Modelling Approaches", International Journal of Chemical Reactor Engineering, 1: A5.

14. Brucato A., Grisafi F., Montante G., 1998, "Particle Drag Coefficients in Turbulent Fluids", Chemical Engineering Science, 53, 3295-3314.

15. Micheletti M., Nikiforaki L., Lee K.C., and Yianneskis M., 2003, "Particle Concentration and Mixing Characteristics of Moderate-to-Dense Solid-Liquid Suspensions", Ind. Eng. Chem. Res, 42, 6236-6249. 